\title{
Design and Application of Information Management System based on ASP.NET
}

\author{
Guang Zhao ${ }^{1, \text { a }}$, Hongyuan Huang ${ }^{2, \text { b }}$ \\ ${ }^{1}$ Sports Information \&Technology Department, Wuhan Sports University, China \\ ${ }^{2}$ Sports Department, Zhongnan University of Economics and Law, China \\ akaixinyezi@126.com, bsmallittlecat@126.com
}

Keywords: Training Information, ASP.NET, ADO.NET

\begin{abstract}
With ASP.NET and ADO.NET technologies the staff training information management system was developed, realize the network office automation, make the information management betimes, and improve the work efficiency. The function peculiarity and design project of the system were discussed deeply, and put forward a new method of ASP.NET application.
\end{abstract}

\section{Introduction}

The system was developed by using tools such as ASP.NET, ADO.NET, C\#, SQL server 2000. Compared with asp, ASP.NET has powerful advantages: First, ASP.NET page need to be compiled once without recompilation, until the page was repaired change or web applications was restarted. It makes the speed to be promoted after much access. Second, ASP.NET supports application real-time update. Third, ASP.NET makes it easier to write code by using "code-behind", and makes Structure more clearly, reduce the complexity of the system development and maintenance.

\section{Organization of the Text}

System technical plan. The system uses the B/S development mode; all programs were installed in training information server. User of Department cans entry and query information by using company Intranet or internet.

ASP.NET system structure model. The system uses the ASP.NET three-layer structure: the presentation layer, business logic layer and data access layer. The presentation layer is responsible for to interact with users, business logic layer is responsible for receiving user input and makes the data from the server will be presented to the customer. Business logic layer is responsible for receiving the request from the browser and will be asked to data access layer, and will be asked to deal with the results to a browser. Among them, Web form is the core of ASP.NET application, it is fundamental to show the customer data and information, response and dealing with customers and display Web form of the generation of interaction of information and data. Data layer provides the data services for logic layer through the ADO.NET manipulate data, such as storage data operation results, return data retrieval results, and so on.

Database access strategies. The two core of ADO.NET component is DataSet and.net Framework in access ADO.NET, ADO.NET using XML to provide open access for data. The ADO.NET design and the.net Framework class in the design of the XML is hand-in-hand; they have the same structure components.

ADO.NET and.NET Framework in the XML class focused on DataSet object. Whether DataSet is file or XML stream, it can use the data source from the XML to fill. No matter what the source of data for the data in the DataSet is, DataSet can write XML to accord with the World Wide Web consortium (W3C), and will include its architecture for XML structure definition language (XSD) architecture. Because of the inherent DataSet serialized format for XML, DataSet is good media in the data communication between layers, which makes DataSet to be the best choice in XML Web services remote way to send data and receiving data structure architecture from the XML Web services. 
.NET Framework is a group component includes Connection, Command, Data Reader and Data Adapter object. DataSet is a core component in the open structure assembly of ADO.NET. It is designed to achieve independence data access from any data source. Therefore, it can be used for a variety of different data sources, data for XML, or used to manage local data of the application program. DataSet contains one or more a collection of Dateable objects, those objects by row and column and the primary key foreign key constraints.

.NET Framework makes it more effective on data manipulation and access. Data access strategy is the application used to storage, retrieval and management data. To keep the application execution efficiency and simplify the data access layer, data access layer of the database using stored procedure manner. It can release resources as soon as possible by using or try catch finally block to a stored procedure call.

\section{Development strategy and structure design of system \\ Development strategy of system}

* The development of this system adopt strategy of overall planning, step-by-step implementation. After overall planning, the system is step-by-step developed in the guidance of overall planning.

* The modular design principle. The structure has good flexibility; the user can accord the actual situation of the expansion module.

* Distribute module by the right of use. The organization structure in the reality and the role in network office will be organic combined well, the reality of the authority of the organization setting is applied directly to the platform power management.

Structure and function of the system. The information management system can be run on Windows 2003 Server and above the operating system platform, Web Server is IIS, database Server is Microsoft-SQL Server2000, development tools using Microsoft Visual Studio, NET2003 and DreamWeaver8, etc. The working process includes:

User login through the permissions judgment, ordinary users can only view, reading and query information. The group company subordinates more training center, and each training center is responsible for the management of the center of personnel training business as a registered users.

Registered users can be completed in addition to ordinary users operating outside; still can this training centre in the information management module information recorded, modification and deletion of operation.

In addition to system management insurance, only registered user has the operation authorization of their entry information. Every training centre can post their own training information, including teaching programs, curriculum and related information, according to attend trained staff training class formed.

By the end of the course, record the trainee results, according to results issued certificate, record certification and verify date, writes down the personnel files for the system will automatically to staff certificates use period for the management, and to have the personnel of certificate of expired on system hint, shows its are in an unit to ensure timely training.

Training information mainly includes training organization's information release, browse, search function; Personnel management including personnel to add, modify and delete etc function; The class of the class management including from formation to the end of each management; The curriculum management including the formation of class of class management and substitute teacher management; The unit of the unit management including the add, modify and delete operation; To maintain the system including the user account management, rights management, etc.

\section{Design details of the system}

Login process. Users login system through the login page by input user name and password correct validation. The system gives the wrong information and prohibit user from entering without verification; or it display the system main frame page, read the system module information and provide module interface. Login system used a stored procedure is as follows: 


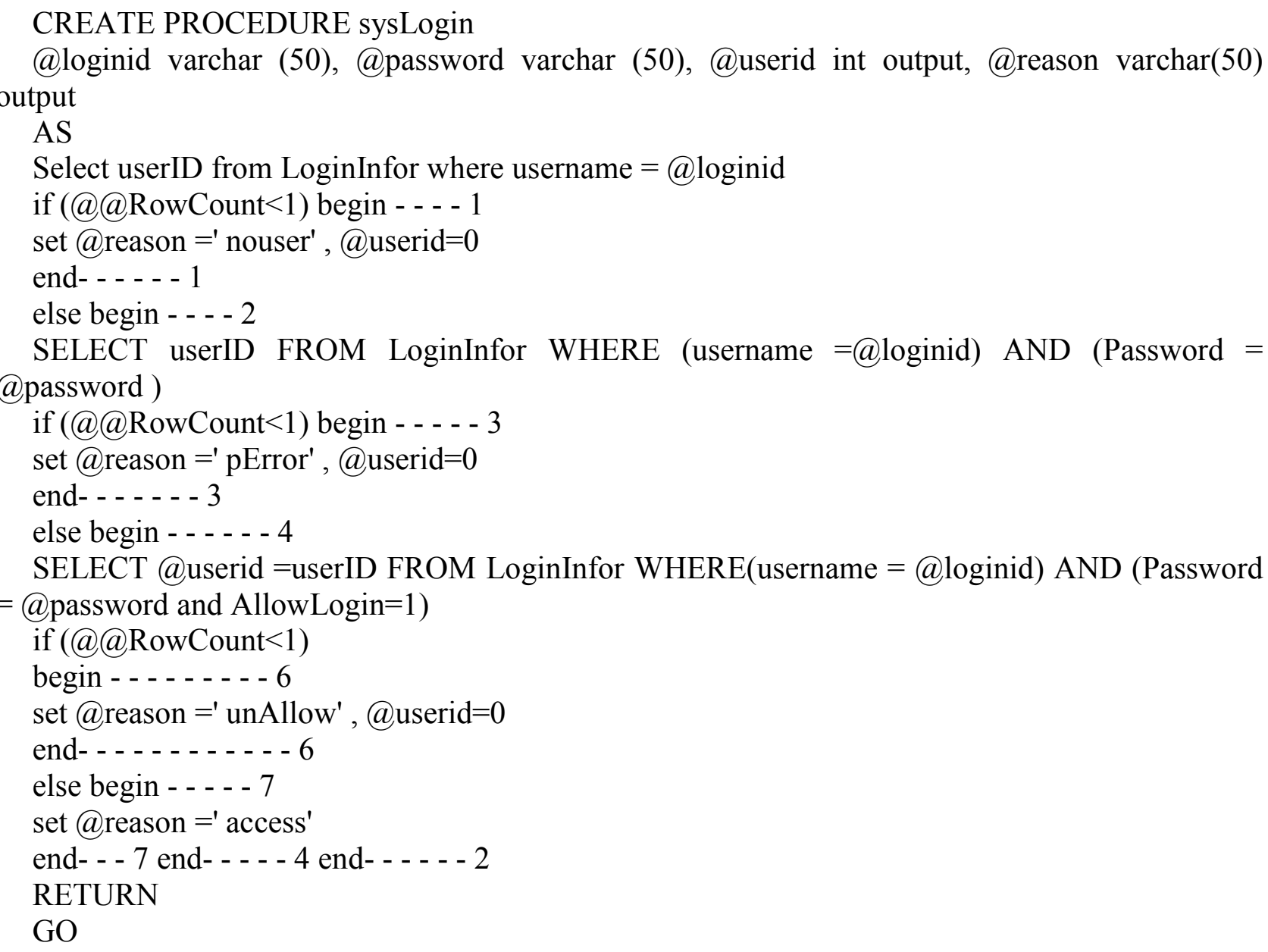

Page rights management. The authority is divided into three levels by the actual management authority distribution principle, the first level is the system administrator; the second level is the training organization's importer; the third level is the general visitors. The training organization's importer assigns authority to the general visitors, and importer permissions by the system administrator distribution. When the page is loaded, system first calls the base class page initialization function, set module name and identity verification.

\section{The conclusion}

This system has a good interface, making users operate more conveniently. The prompting information makes users to be aware of their operation. The inputting information processing (including entry, modify, delete) will be shown immediately on the page corresponding columns. The system Installation can be only accomplished on the server, all of the system management and upgrading can be done only on the server side, and the client does not need any set change. System upgrading and updating does not affect the user's data.

It makes the management more efficient that to use new information management system based asp.net in company circumstance, which is a new innovation of asp.net application. From the overall system architecture to data access strategies, it is used in more advanced processing method, program design for more reasonable and convenient. 


\section{References}

[1] R. Rivest,A. Shamir, L. Aldeman, “A Methoed for Obtaining DigitalSignatures and Public-key Cryptosystems,'J. Communications of the ACM, 1978, 21(2): 120-126.

[2] A .Fiat, "Batch RSA,"C. Proc of Crypto '89, LNCS435, 1989.Berlin: Springer -Verlag, 1989:175-185.

[3] D.Boneh, H.Shacham, "Fast Variants of RSA,"R.RSA Laboratories Cryptophytes, 2002,5(1):1-8.

[4] T. Takagi. Fast RSA-type cryptosystem modulo pkq. In H. Krawczyk, editor, CRYPTO, volume 1462 of Lecture Notes in Computer Science, pages 318-326. Springer, 1998.

[5] T.Matsumoto, K.Kato, "Speeding up secret computations with insecure auxiliary device,"C.Proc of the 8th Annual International Crypto Conference on Advances in Cryptology.London: Springer-Verlag, 1988.

[6] C.Castelluccia, E. Mykletun, and G. Tsudik. "Improving secure server performance by re-balancing SSL/TLS handshakes,"C. Proc of the 2006 ACM Symposium on Information, computer and communications security.New York: ACM, 2006: pages 26-34.

[7] J-J. Quisquater and C. Couvreur, "Fast decipherment algorithm for RSA public-key cryptosystem,'J. Eletronic Letters, vol 18:905-907, 1982. 